\title{
A Numerical Study of the Performance of a Contoured Shock Tube for Needle-free Drug Delivery
}

\author{
Md. Alim Iftekhar Rasel* and Heuy Dong $\mathrm{Kim}^{\dagger}$
}

\begin{abstract}
In recent years a unique drug delivery system named as the transdermal drug delivery system has been developed which can deliver drug particles to the human skin without using any external needle. The solid drug particles are accelerated by means of high speed gas flow through a shock tube imparting enough momentum so that particles can penetrate through the outer layer of the skin. Different systems have been tried and tested in order to make it more convenient for clinical use. One of them is the contoured shock tube system (CST). The contoured shock tube consists of a classical shock tube connected with a correctly expanded supersonic nozzle. A set of bursting membrane are placed upstream of the nozzle section which retains the drug particle as well as initiates the gas flow (act as a diaphragm in a shock tube). The key feature of the CST system is it can deliver particles with a controllable velocity and spatial distribution. The flow dynamics of the contoured shock tube is analyzed numerically using computational fluid dynamics (CFD). To validate the numerical approach pressure histories in different sections on the CST are compared with the experimental results. The key features of the flow field have been studied and analyzed in details. To investigate the performance of the CST system flow behavior through the shock tube under different operating conditions are also observed.
\end{abstract}

Key Words: Vaccines, Contoured shock tube, Transient, Supersonic nozzle, Gas particle dynamics, Biolistics, Drugs.

\section{Introduction}

Delivering drug to the human body is a very important part of medical science. With the advancement of the technology different systems have been established to do it properly. For the last few decades plastic syringe are widely used for this purpose. But in recent times transdermal drug delivery, a unique drug delivery system has been developed which delivers drug particle to the human skin without using any kind of external needle. The particles are accelerated by high speed gas flow to penetrate the outer layer of the skin and have a pharmaceutical effect.

'Department of Mechanical Engineering, Andong National University, Korea

E-mail : kimhd@andong.ac.kr

*Department of Mechanical Engineering, Andong National University, Korea
The trandermal drug delivery system was pioneered by Sanford et al. who designed a system to deliver DNA coated metal particles into plant cells for genetic modification ${ }^{(1)}$. Later it was modified by Bellhouse et al for human treatment with particle accelerated by entrainment in a supersonic gas flow ${ }^{(1)}$. Recently, Kendall designed different systems and studied them both experimentally and numerically to make it more convenient for clinical use ${ }^{(1,2,3,4,5)}$.

Convergent divergent supersonic nozzle (CDSN) is the first drug delivery system designed by Kendall et al ${ }^{(1)}$. To analyze the performance of the device it has been studied numerically and experimentally ${ }^{(1,2,3)}$. Later it is modified and the contoured shock tube is designed.

The transdermal drug delivery offers lots of advantages over the conventional system. The whole process is painless and injury free. As there is no external needle, no sharp is exposed for contamination hazard. The delivering process is also very easy and 
there is no need of any skilled operator.

To get the optimum effect of the delivered drug it is very important to deliver it into the desired layer of the skin. By controlling the particle velocity it is possible to do so by transdermal drug delivery system. It also offers exciting prospect for some unique treatments like DNA vaccination, anti-viral drug delivery which are difficult to execute with the conventional technology. As the drugs are delivered in powder form there is no need to store it in liquid form anymore. That makes storages and transportation much easier therefore making it less costly.

The performance of the contoured shock tube has been studied extensively ${ }^{(3,4)}$. For proper delivery of drug particle it is important to deliver it with a uniform velocity and spatial distribution something which the previous devices failed to provide. But CST system seems to produce uniform velocity and also with spatial distribution. The impact of the microparticle to the human skin, porcine skin and canine mucosa are investigated along with the biological response ${ }^{(10,11,12)}$.

This paper aims at analyzing the flow dynamics through a contoured shock tube as well as observing the behavior under different operating conditions. As it is very hard to do so experimentally, numerical approach is taken using computational fluid dynamics. Fluent 14, a commercial software for CFD applications is used for flow simulation and analysis.

The contoured shock tube system is introduced at the beginning of the paper followed by the details description of the numerical approach taken to simulate the flow field. The numerical results are compared with the experimental results to validate the numerical approach and to analyze the flow.

The shock tube is used to allow the formation of the primary shock, the contact surface and also to increase the separation between the particle cloud and the primary shock. The divergent nozzle section is included to further accelerate helium to a correctly expanded supersonic exit condition.

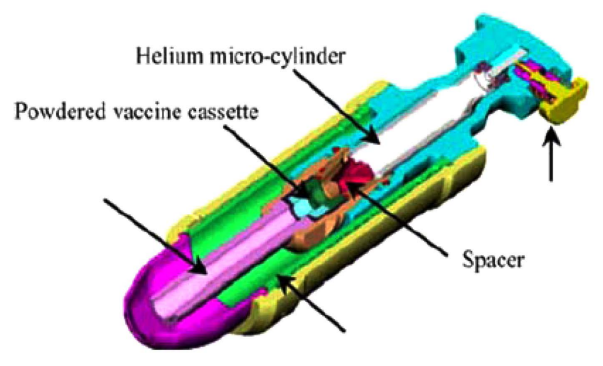

Fig. 1. The contoured shock tube.

\section{The contoured shock tube (CST) system}

A standard CST device for clinical use is shown in the Fig. 1 showing the important components. The main components of the CST device are the microcylinder, the drug cassette, a contoured shock tube and a divergent nozzle section. Compressed helium at a high pressure is stored in the micro-cylinder. The drug cassette retains the drug particles between a set of bursting membrane which act as diaphragms in a classical shock tube. Key aspects of the CST systems are discussed in details computationally and experimentally ${ }^{(5,6)}$.

The helium is stored at a very high pressure (4 MPa) in the micro-cylinder. Two polycarbonate diaphragm of $20 \mu \mathrm{m}$ thickness are loaded with a powdered particle load of $1.1 \pm 0.1 \mathrm{mg}$. When the helium is released from the micro-cylinder it mixes with the residual air in the rupture chamber simultaneously increasing the pressure. After a specific pressure difference is reached the diaphragm ruptures and a primary shock is formed which propagate through the shock tube. As the primary shock flows through the shock tube to the nozzle exit it creates a starting process followed by a quasi-steady supersonic flow (QSSF). In course of this processes particles are accelerated to the nozzle exit.

Figure 2 shows the CST system under experimental and numerical study with the sensor locations for the static pressure measurement on the shock tube wall. A pre-filled gas chamber is considered instead of a micro-cylinder and the drug cassette is modeled by a 


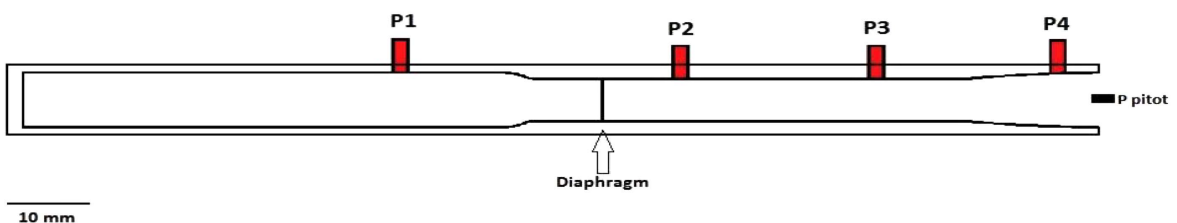

Fig. 2. The CST system under numerical study with the sensor locations.

single diaphragm. No silencer is considered for numerical modeling.

\section{Numerical Simulation}

\subsection{Governing Equations}

The flow physics was mathematically modeled using unsteady Reynolds Averaged Navier-Stokes equations. The governing equations in the Cartesian form can be written as,

Continuity,

$\frac{\partial \rho}{\partial t}+\frac{\partial}{\partial x_{i}}\left(\rho u_{i}\right)=0$

Momentum,

$$
\begin{gathered}
\frac{\partial}{\partial t}\left(\rho u_{i}\right)+\frac{\partial}{\partial x_{i}}\left(\rho u_{i} u_{j}\right)=-\frac{\partial p}{\partial x_{i}}+\frac{\partial}{\partial x_{j}} \\
{\left[\mu\left(\frac{\partial u_{i}}{\partial x_{j}}+\frac{\partial u_{j}}{\partial x_{i}}-\frac{2}{3} \delta_{i j} \frac{\delta u_{i}}{\delta x_{i}}\right)\right]+\frac{\partial}{\partial u_{j}}\left(-\rho \overline{u_{i}^{\prime} u_{j}}\right)}
\end{gathered}
$$

Energy,

$$
\frac{\partial \rho E}{\partial t}+\frac{\partial}{\partial x_{i}}\left[u_{i}(\rho E+P)\right]=\frac{\delta}{\partial x_{i}}\left[\left(\alpha+\frac{C_{p} \mu_{t}}{P_{r t}}\right) \frac{\partial T}{\partial x_{i}}+u_{j}\left(\tau_{i j}\right)_{e f f}\right]
$$

SST k- $\omega$ was used as the turbulence model. In this model the turbulent viscosity is calculated by solving the transport equation for turbulent kinetic energy $(\mathrm{k})$ and the specific dissipation rate $(\omega)$ as shown in the below equation.

$$
\begin{aligned}
& \frac{\partial \rho k}{\partial t}+\frac{\partial}{\partial x_{t}}\left(\rho k u_{i}\right)=\frac{\delta}{\partial x j}\left(\Gamma_{k} \frac{\partial k}{\partial x_{j}}\right)+G_{k}-Y_{k} \\
& \frac{\partial \rho \omega}{\partial t}+\frac{\partial}{\partial x_{t}}\left(\rho \omega u_{i}\right)=\frac{\delta}{\partial x j}\left(\Gamma_{\omega} \frac{\partial \omega}{\partial x_{j}}\right)+G_{\omega}-Y_{\omega}+D_{\omega}
\end{aligned}
$$

At the beginning the high pressure driver section is assumed to be filled with a mixture of helium and residual atmospheric air. The mass fraction distribution of different species involved was modeled using species transport equations.

$$
\frac{\partial \rho Y_{i}}{\partial t}+\nabla\left(\rho u_{i} Y_{i}\right)=-\nabla j_{i}+R_{i}+S_{i}
$$

It predicts the local mass fraction of each species $Y_{i}$. Here $R_{i}$ is the net rate of production of species by chemical reaction and $S_{i}$ is the rate of creation by addition from the dispersed phase plus any user defined sources.

\subsection{Numerical Scheme Used}

Fluent 14, a commercial CFD application software is chosen to numerically simulate the flow field. Ideal gas behavior was considered for all the species and the viscosity variation with respect to temperature was modeled using Sutherland viscosity model. The flux component of the governing equation was discretized using upwinding Roe vector different splitter scheme. A second order implicit scheme was used for the temporal discretization. The governing flow equations were solved in a coupled manner. An overall second order scheme is satisfied spatially and temporally.

The experimental setup is circular therefore axissymmetric model is used in Fluent to get more accurate results. Only one diaphragm is modeled instead of two in numerical model to avoid complexity. Also the non ideal diaphragm rupture process is not considered. As we are only interested in the events after the diaphragm rupture a pre-filled driver section (mixture of helium and local atmospheric air) is assumed instead of simulating the filling process. The averaged diaphragm rupture pressure is taken as $1550 \mathrm{kPa}$ in the numerical modeling. The particle effect is not considered.

\subsection{Computational Grid and Boundary Condition}

Figure 3 shows the complete computational domain 


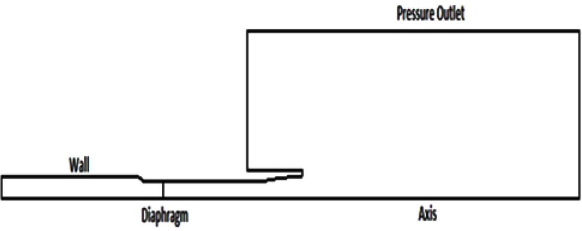

Fig. 3. Computational domain with boundary conditions.

for the numerical study. The boundary conditions are also shown in specific locations. The domain is extended to $15 \mathrm{D} \times 4 \mathrm{D}$ where $\mathrm{D}$ is the diameter of the nozzle exit plane. The grid has 158960 cells with 1,004 points in the axial and 90 points in the radial direction. The rest are distributed in the downstream section of the nozzle exit.

A pre-filled driver section at a very high pressure $(1,550 \mathrm{kPa})$ is considered as mentioned earlier. The diaphragm boundary is instantly removed which leads to the formation of a shock wave. Atmospheric pressure is specified in the driven section and the outlet

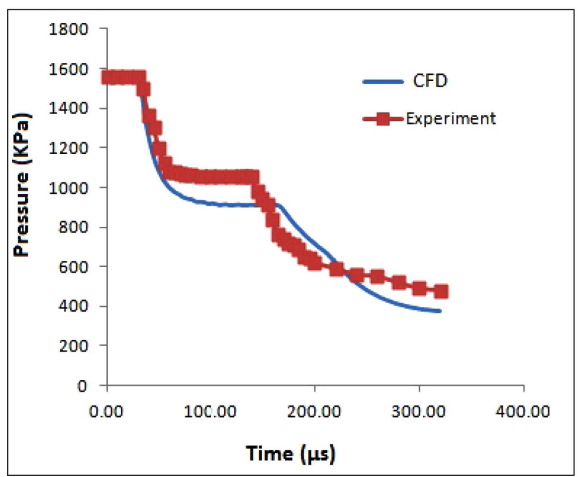

(a)

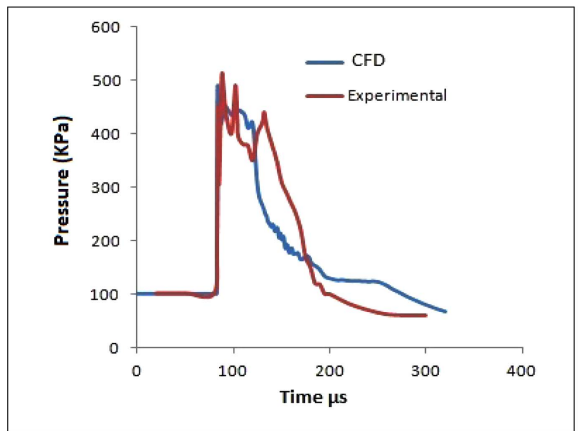

(c) boundary. The wall temperature is assumed to be constant with no slip condition.

\section{Results and Discussion}

Numerical static pressure histories measured in different section of the CST device are compared with the experimental results. The experimental results are obtained considering the polystyrene micro particles with $39 \mu \mathrm{m}$ diameter whereas the numerical model does not consider any particle.

Figure 4 demonstrates the numerical and experimental pressure histories in different section of the CST system along with the Mach number history in the nozzle centre. The pressure history in the driver section (P1) is shown in Fig. 4(a). The pressure remains constant for some times then suddenly starts to decay. Initially the experimental pressure values are a bit higher than the numerical values. This discrepancy is because of

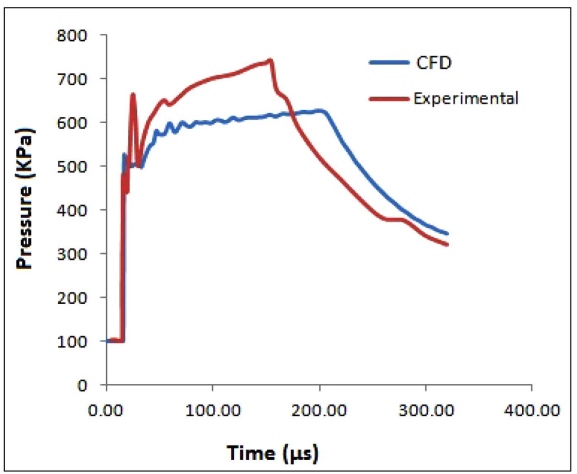

(b)

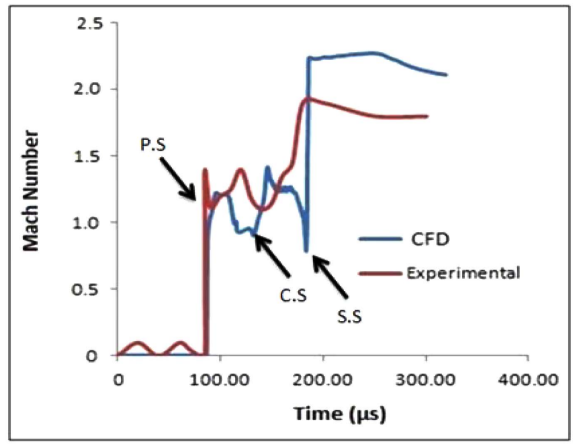

(d)

Fig. 4. Experimental and numerical pressure histories in position (a) P1, (b) P2, (c) P4 and the (d) Mach number histories in the centre of the nozzle exit. 
the assumption of a pre-filled gas chamber instead of considering micro-cylinder. The effect of microcylinder was investigated by Marrion et al and found to have negligible effect on the nozzle flow ${ }^{(9)}$.

Figure 4 (b) shows the pressure histories in the shock tube section (P2). Like the driver section (P1), P2 also shows the same kind of discrepancy and because of the same reason.

Figure 4 (c) shows the pressure histories in the nozzle section (P4). For many reasons the nozzle section is very important in the CST device. The divergence section ensures that the particles are delivered in a larger area. For the first generation needle-free devices like the CDSN system the nozzle section creates undesirable secondary shocks and flow separation which reduces the efficiency of the device to a great extent. The numerical and the experimental pressure histories are in good agreement. The typical starting process in a supersonic nozzle is very clear in the Fig. $4^{(8)}$. The starting process is followed by a Quasi-steady supersonic flow (QSSF). The numerical result shows that the starting process starts a bit early than the experimental results. This discrepancy is also because of not considering the helium micro-cylinder. Neglecting the particle effect also shows some effect as the numerical value looks to cross the experimental values just before the QSSF starts. The assumption of ideal diaphragm rupture may also have some effect.

Figure 4 (d) shows the Mach number histories on the centre of the CST exit plane which gives a clear idea about the events inside the contoured shock tube. The primary shock, contact surface and the secondary shock are clearly indicated along with their duration. The staring process and the QSSF are also very clear and are in agreement with experimental results.

Overall the numerical and the experimental results show quiet good agreement with the experimental results with slight discrepancy. The discrepancy is because of three reasons. They are not considering the micro cylinder, neglecting the particle effect and the assumption of ideal diaphragm rupture. This indicates that the discrepancy is not because of numerical error

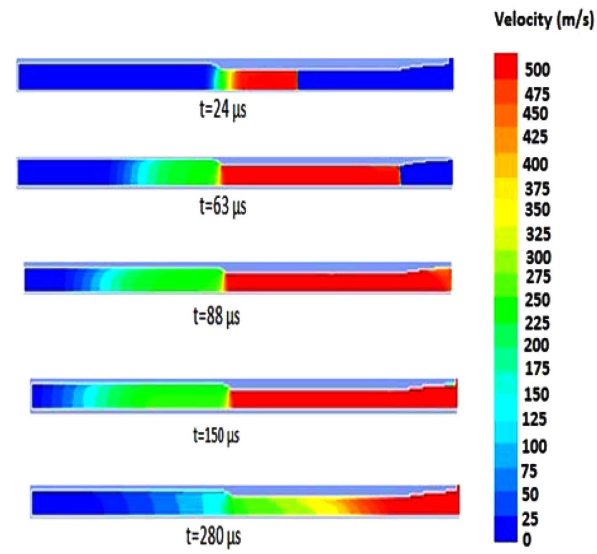

Fig. 5. Simulated velocity contour at different time.

rather because of the method implemented.

To get a visual demonstration of the flow phenomena through the contoured shock tube velocity contours at different times are shown in Fig. 5. The primary shock is clearly seen which is moving through the shock tube from left to right. To show the formation of primary shock more clearly temperature contour just after the diaphragm rupture is shown indicating the shock front, contact surface and the expansion wave in Fig. 6.

The velocity contour after $152 \mu$ s and $280 \mu$ s shows respectably the nozzle starting process and the quasisteady supersonic flow. To further analyze the starting process and the QSSF velocity, density and Mach number contours are shown after $152 \mu$ s and $280 \mu \mathrm{s}$ from the diaphragm rupture in Fig. 8 and 9. In Fig. 8 (a) the secondary shock is clearly seen as it demonstrates a sharp velocity drop. This is more clear in the Mach

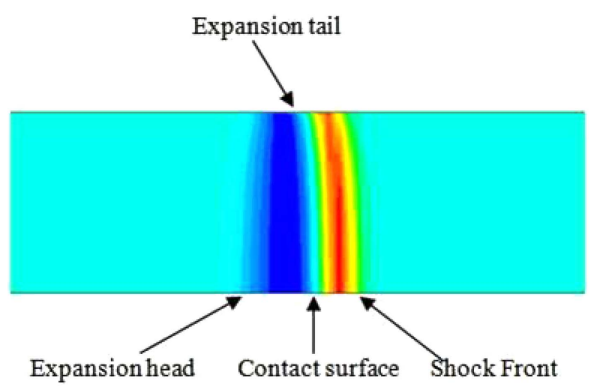

Fig. 6. The formation of the primary shock. 


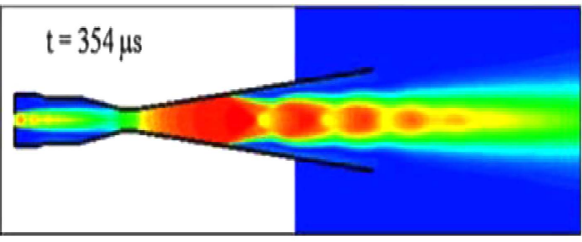

Fig. 7. Simulated velocity contour $354 \mu$ s after the diaphragm rupture.

number contour in Fig. 8(b). As mentioned earlier secondary shock used to create flow separation in the first generation drug delivery devices as shown in Fig. $7^{(3)}$. But no such kind of separation is seen in Fig. 8 (a) or 8 (b). Figure 9 shows the velocity and the density contour $280 \mu$ s after the diaphragm rupture demonstrating the QSSF. For this case also flow separation or any other kind of undesired shock is not observed.

To investigate the effect of different rupture pressure in the flow field the flow phenomena has been simulated assuming three different rupture pressure 2,250 KPa, $1,550 \mathrm{KPa}$ and $1,000 \mathrm{KPa}$. The pressure histories in the driver and the nozzle section are shown in Fig.10.

Figure 10 (a) demonstrates the pressure histories in the driver section (P1) at different rupture pressure. For the three cases the initial pressure remains constant for some times and suddenly starts to decay.
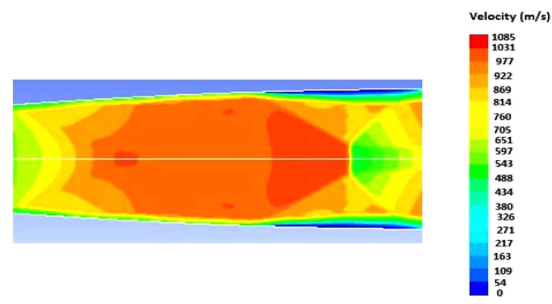

(a)

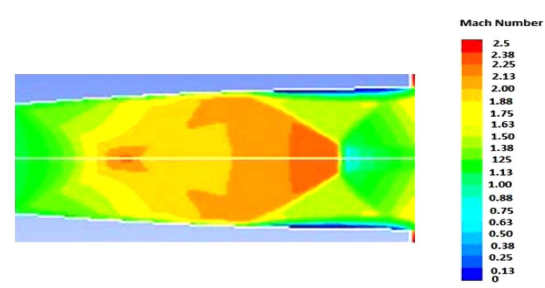

(b)

Fig. 8. Velocity and Mach number contour $150 \mu$ s after the diaphragm rupture.
The decay process seems to be quicker in the higher rupture pressure process.

Figure 10 (b) demonstrates the pressure histories at different rupture pressure in the nozzle section. The

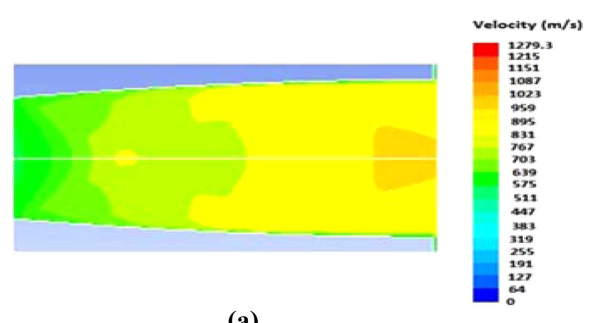

(a)

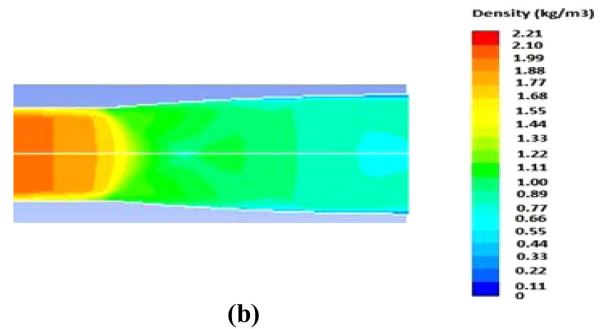

(b)

Fig. 9. Simulated velocity and density contour $280 \mu \mathrm{s}$ after the diaphragm rupture.

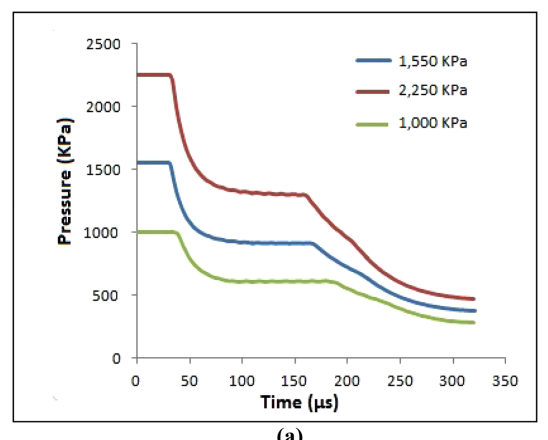

(a)

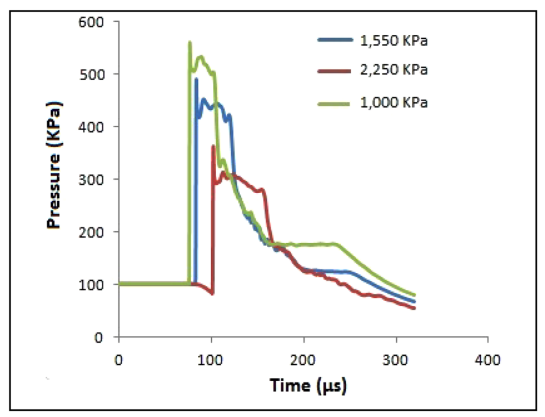

Fig. 10. Pressure histories in the (a) driver (P1) and the (b) nozzle section in different rupture pressure. 
typical starting process along with the QSSF is very clear in all the three cases indicating a rapid pressure drop. As rupture pressure increases, the duration the starting process seems to decrease. The starting of the QSSF is also quicker in the high rupture pressure cases with higher pressure values. One of the priorities in the CST system is to try and accelerate most number of particles in the QSSF not in the starting process.

\section{Conclusion}

The flow phenomena through a contoured shock tube for needle-free drug delivery has been investigated, analyzed and explained in details along with its behavior under different operating conditions. A commercial CFD application software Fluent 14 has been used to simulate and analyze the flow field. Numerical static pressure histories in different sections of the CST device are compared with the experimental results and are found in good agreement. Later these pressure histories are used to analyze the flow field. The behavior of the secondary shock was thoroughly investigated comparing it with the first generation drug delivery devices. Flow separation or any other unwanted shock is not observed.

To investigate the performance of the contoured shock tube pressure histories in the driver (P1) and the nozzle section (P4) under different rupture pressure are investigated. A more rapid starting process is observed in the higher rupture pressure. The QSSF is also quicker with the increase of rupture pressure.

\section{Acknowledgement}

This work was supported by the National Research Foundation of Korea (NRF) grant funded by the Korea government (MEST) (2011-0017506).

\section{References}

1) M.A.F Kendall, N. J. Quinlan, S.J. Thorpe, R.W. Anisworth, B.J Bellhouse, 2004, "Measurements of the gas and particle flow within a converging- diverging nozzle for high speed powdered vaccine and drug delivery", Experiments in Fluids, Vol. 37, pp. 128-136.

2) N. J. Quinlan, M.A.F Kendall, B.J Bellhouse, R.W. Anisworth, 2001, "Investigation of gas and particle dynamics in first generation needle-free drug delivery device", Shock Wave, Vol. 10, pp. 395-404.

3) Yi Liu, 2006, "Performance studies of particle acceleration for transdermal drug delivery, 2006", Med Bio Comput, Vol. 44, pp. 551-559.

4) M.A.F Kendall, 2002, "The delivery of particulate vaccines and drugs to human skin with a practical, handheld shock tube-based system", Shock Wave, Vol. 12, pp. 23-30.

5) Y. Liu, M. A. F. Kendall, 2006, "Numerical analysis of gas and micro-particle interactions in a hand-held shock-tube device", Biomed Microdevices, Vol. 8, pp. 341-351.

6) Y. Liu, M. A. F. Kendall, N. K. Truong, B. J. Bellhouse, 2002, "Numerical and Experimental analysis of a high speed needle-free powdered vaccines delivery device", 20th AIAA Applied Aerodynamics Conference 24-26 June 2002, St. Louis, Missouri.

7) R.F. Chisnell, 1957, "The motion of a shock wave in a channel, with applications to cylindrical and spherical shock waves", Journal Fluid Mechanics, Vol. 2, pp. 286-298.

8) C. E. Smith, 1966, "The starting process in a hypersonic nozzle", J. Fluid Mech., 24(4), pp. 625-641.

9) M. Marrion, Y. Liu, and M.A.F. Kendall, 2005, "The gas-dynamic effects of a hemisphere-cylinder obstacle in a shock-tube driver", Experiments in Fluids, Vol. 38, pp. 319-327.

10) M.A.F Kendall, TJ Mitchell and P. Wrighton-Smith, 2004, "Intradermal ballistic delivery of micro-particles into excised human skin for drug and vaccine application”, Journal of Biomechanics, 37(11), p. 1733-1741.

11) T.J. Mitchell, M.A.F.Kendall, B.J. Bellhouse, "A ballistic study of microparticle penetration to the oral Mucosa,2003", International Journal of Impact Engineering, Vol. 28, pp. 581-599.

12) P.A. Raju, N.K Truong, M.A.F. Kendall, 2006, “(in press) Assessment of epidermal cell viability by Near Infra-Red Multi-photon Microscopy following ballistic delivery of gold micro-particles", Vaccines 24(21), pp. 4644-4647. 